\title{
Microwave assisted pyrolysis of plastic waste for production of fuels: a review
}

\author{
Haroon Arshad ${ }^{1,}{ }^{*}$, Shaharin A Sulaiman ${ }^{1}$, Zahid Hussain ${ }^{2}$, Yasin $\mathrm{Naz}^{3}$, Firdaus Basrawi ${ }^{4}$ \\ ${ }^{1}$ Department of Mechanical Engineering, Universiti Teknologi PETRONAS, 32610 Seri Iskandar, \\ Malaysia \\ ${ }^{2}$ Department of Chemistry, Abdul Wali Khan University, 23200 Mardan, Pakistan \\ ${ }^{3}$ Department of Physics, University of Agriculture, 38040 Faisalabad, Pakistan \\ ${ }^{4}$ Department of Mechanical Engineering, Universiti Malaysia Pahang, 26600 Pekan, Malaysia
}

\begin{abstract}
This paper presents an overview of advantages of microwave assisted pyrolysis of waste plastics together with its limitations. It has been established that microwave induced pyrolysis can be used to get value added chemicals and fuels through its numerous noted advantages in contrast to conventional pyrolysis. The process has the potential for fast, volumetric and selective heating of plastics for the recovery of energy. The limitation in the use of dielectric material as absorbent in plastic pyrolysis has been highlighted. Special focus has been given to the constraints encountered in the accurate measurement of temperature and uniform heating in microwave assisted pyrolysis. A new alternative method based on microwave-metal interaction in the pyrolysis of plastic waste has been presented. Further it has been realized that proper investigation and understanding of microwave process shortcomings is fundamental to the successful implementation of the technology and at the same time provide a sustainable environment in the endeavor for waste to energy mission.
\end{abstract}

\section{Introduction}

Plastics are included among indispensable items of modern times. It has diverse applications due to its many excellent. Common types of plastics found in municipal solid waste (MSW) comprise high density polyethylene (HDPE), low density polyethylene (LDPE), polypropylene (PP), polystyrene (PS), polyethylene-terephthalate (PET) and polyvinyl chloride (PVC) [1], which are the principal contributors to the non-biodegradable class of waste. With rapid growth in the world population, there has been a drastic increase in the use of commodity plastics dominant in packaging, clothing, beverage and trash containers, and numerous other household items. It was observed that the global production of plastics rose to 229 million in 2013 with a $4 \%$ gain over that of 2012 [2]. At the same time, there was also huge accumulation of waste due to continuous rise in demand of plastics each year [3]. It was reported that the plastic waste reached to over 33 million tons in US according to 2013 statistic [4]. Moreover, in Europe a study revealed that a total of 25 million tons of plastics went to the wastestream in 2012. Further, it was found that $38 \%$ of

\footnotetext{
${ }^{*}$ Corresponding author: haroonarshad00@gmail.com
} 
this waste was disposed to landfill, $26 \%$ were recycled and the remaining $36 \%$ was utilized for energy recovery [2]. It was also reported that plasticizers (additives used to enhance plastic properties) found in some specific plastics like PVC can also leach out from improperly designed landfills and may contaminate groundwater which is caused by the production of phthalate esters or PAEs [5]. Further it was explored in a study that open dumping of municipal solid waste poses environmental hazards through the transfer of heavy metals to the soil which adversely affect our vegetation [6]. Another problem was identified associated with disposal of plastic waste into oceans which results in formation of soap and garbage patch like the great Pacific garbage patch posing risk to health of aquatic animals [7]. Moreover, incineration of plastic waste may lead to environmental pollution through the toxic emissions [8].

Alternatively, the disposal of plastic waste through recycling has been considered as an unattractive option due to the extra cost involved in its recovery and intensive requirement of labor in the separation of its components [3]. The limitations on recycling method and ever-increasing load on plastic waste has created a need for the development of alternative technologies to solve the problems associated with disposal. Greater interest was observed on the conversion of waste plastic into value added chemicals and fuel products in a range of published papers on conventional as well as microwave assisted pyrolysis [3], [7], [912]. The conventional method also known as traditional method converts waste into fuel products through thermal or catalytic pyrolysis. Thermal pyrolysis which is uncatalyzed gives low quality products over a wide range [13]. However, application of suitable catalysts in catalytic pyrolysis has the potential to narrow the range of final products leading to outstanding selectivity for getting valuable products at lower temperatures [14]. Pyrolysis is a process that involves thermochemical breakdown of organic material at elevated temperatures $300-900^{\circ} \mathrm{C}$ in oxygen free environment, resulting in oil and gas yields with char as the solid residue. Based on heating strategies, pyrolysis can be classified as conventional pyrolysis and microwave assisted pyrolysis. The former involves surface heating of plastic waste using fuels like coal, whereas the latter works on the principle of microwave electromagnetic radiations conversion to thermal energy and has the potential for volumetric heating which is faster as compared to surface heating.

Though there are many advantages in microwave heating, there are also certain limitations. Accurate measurement of temperature within the reaction mixture is difficult [15]. Moreover, uneven distribution of scattered hotspots within the sample may result in non-uniform heating according to a simulation study on microwave design [16]. Another challenge has been the quantification of dielectric properties of the material to be treated since heating efficiency depends strongly on dielectric response of materials and there is insufficient data on the dielectric materials to date [3]. Problems related to microwavemetal discharges and scale-up of technology are elaborated. An alternative method of microwave-assisted pyrolysis of plastics and its future challenges have been highlighted. Therefore, this review focuses on the shortcomings of microwave assisted pyrolysis together with its advantages in contrast to conventional heating. The objective of this paper is to broaden the understanding of the limitations of microwave processing which is crucial to the success of any proposed work wherein relevant considerations could be made to get the desired results like efficient heating, accurate temperature measurement, minimum energy input and overall reduced cost. 


\section{Microwave heating technology}

\subsection{Microwave and conventional heating}

Microwave is an electromagnetic form of energy found in the range of wavelengths from 0.001-1 m operating at a frequency band of 0.3-300 GHz. Most of the common microwaves used, particularly the domestic ovens operate at frequency of $2.45 \mathrm{GHz}$. Since microwaves have the ability to penetrate through solid material, it can accomplish volumetric heating in contrast to conventional method of heating in which only surface heating is achievable. The heat flow patterns showing the temperature distribution produced during the two heating schemes is illustrated in Fig. 1 [17]. The major difference between the microwave and conventional heating is based on the heat transfer mechanism. Conventional methods utilize furnaces to initially heat the surface, before any conductive heat transfer towards the inner core of the material takes place [18].
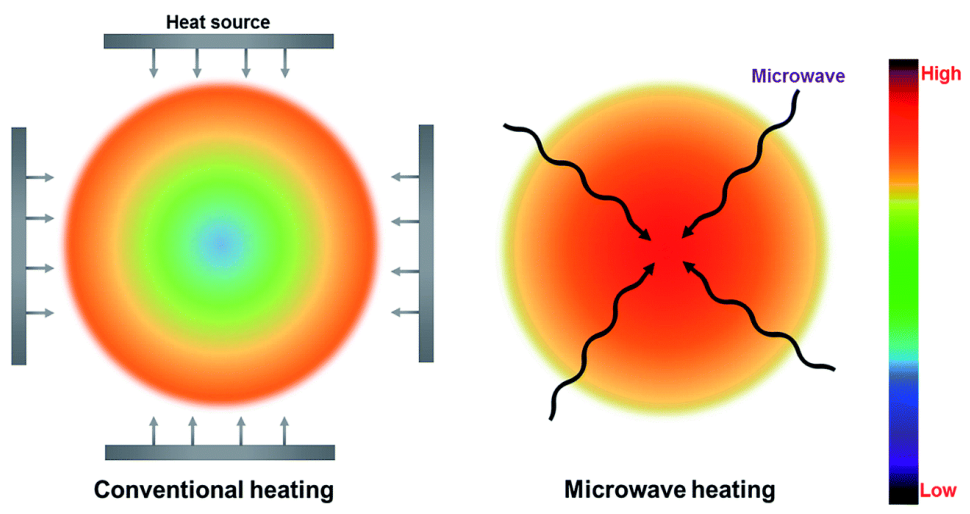

Fig. 1. Microwave and conventional heating patterns [17].

In case of microwave assisted pyrolysis of plastics, additional use of dielectric material called as absorbent is required. A study conducted on pyrolysis of plastic waste utilized carbon black as absorbent to convert the microwave energy to heat required for pyrolysis [12]. Contrarily, conventional heating does not need any absorbent due to direct mode of heat transfer to the material. Another criterion used to differentiate between microwave and conventional pyrolysis techniques is the distribution of products. An investigation led by (Khaghanikavkani, 2013) on variation in product composition was carried out for pyrolysis of plastics using microwaves and conventional method. The results revealed a very similar distribution of pyrolysis products. However, the heating uniformity was found to be considerably improved in contrast to conventional method as a result of microwave volumetric heating leading to faster pyrolysis. In case of plastics at a suitable scale $(0.5 \mathrm{~kg})$, the microwave heating has proved to be an efficient and cleaner process with faster and easier control [19].

There are several benefits of microwave heating in addition to volumetric heating [20], [21]. Selective material heating is a unique advantage of this technology that targets the specific material. In this process, the magnitude of heating strongly depends on the dielectric strength of the material. Since plastics have poor dielectric strength, they are mixed with dielectric absorbents like carbon which has been observed to convert microwave energy into thermal energy in a short span of time [22]. Thus, heating efficiency may vary for different materials which has also been a great challenge to industries [3]. Here, rapid heating can be easily promoted. It was pointed out that decomposition temperature up to $450^{\circ} \mathrm{C}$ in solid 
organic polymers can be achieved in a fraction of the time taken in conventional pyrolysis [23]. High temperatures can be reached in few minutes rather than hours in most cases, when the materials are heated at higher heating rates [23]. Another unique feature of this method is the non-contact heating. If microwaves can be controlled properly, it has the potential to treat the material without any physical contact with the heated sample [23]. Additionally, the control of the process is also simple. Plastic pyrolysis was achieved through efficient coupling and easy control of temperature by tuning and power control [24]. Microwaves have also been found to be effective for the upgrading of in-situ pyrolytic vapors into enhanced bio-oils, during biomass pyrolysis $[25,26]$. Many studies have reported faster heating and in situ treatment of waste [22], [27-29]. The flexibility of operation and portability of equipment are the noted benefits of microwave technology [27, 28].

\subsection{Limitations in microwave assisted pyrolysis}

The potential of dielectric materials called as absorbents or susceptors to be used in the pyrolysis of polymers and plastics has been explored widely [22, 27, 28]. However, specific role of susceptor in the energy transfer or catalyzing effect on pyrolysis reaction is still not well known [15]. The effective use of dielectric material at industrial scale pyrolysis is a challenge since heating efficiency differs for different absorbents [3]. It is to be pointed out that not all materials have the ability to absorb microwaves [23]. For example, plastics have low dielectric constant and the mixture with carbon as microwave absorber during pyrolysis may enhance the energy absorbed to be converted to heat in shorter time [22]. Here, the challenge has been the quantification of properties of the dielectric materials to be treated since heating efficiency depends strongly on these properties and there is insufficient data on the dielectric materials to date [3].

To date, macroscopic temperature measurement methods, can only measure the overall or average value of temperature of the heating medium due to detection limitation of measurement devices at temporal and spatial scales, and it has been very difficult to measure temperature of isolated hotspots [30]. With the use of advanced detecting instruments like high-speed camera and optical temperature probe, existence of hotspot effect has been confirmed [31,32]. Another difficulty is faced during accurate measurement of temperature within the reaction mixture. In a study led by Undri et al. [33], on microwave assisted pyrolysis of silicon oil, a temperature lag was observed in IR-based sensors in contrast to conventional use of thermocouples. The temperature monitoring of the process becomes difficult since the temperature goes beyond $1000^{\circ} \mathrm{C}$ and an instrument like thermocouple will melt down. In this scenario, there are two alternatives available for temperature measurement. One is the use of infrared thermometer and the other one being fiber optic thermometer. The infrared thermometer works without contact over a wide range of temperatures. But the IR thermometer sensors can only measure the surface temperature of the reactor which is lower than the temperature of the reaction mixture [15]. In this context only few studies have been able to measure the temperature within the reaction mixture [35-37]. On the other hand, the use of fiber optic device is not possible since it can measure temperatures only up to $400^{\circ} \mathrm{C}$ [38]. But there is a new variant of optical fiber temperature probe which is made of sapphire crystalline fiber and has been used effectively in microwave pyrolysis [39]. In another published work, a thermocouple was used for temperature measurement inside a domestic microwave oven operating under low power of magnetron [15]. This facilitated measurement of temperature inside the reaction mixture during the off-time which is greater than the on-time period in contrast to high power microwave. It was pointed that conventional thermocouple may lead to sparks and trips due to reflection of microwave. Therefore, in this experiment, a chromel-alumel thermocouple was used as a modified version of conventional thermocouple. 
Formation of hotspot or micro-plasmas is another limitation that has been identified as a form of thermal instability associated with rapid heating in a microwave. According to a study, hotspot formation is a result of non-linear dependence of electromagnetic field on the thermal properties of the material [40]. The presence of such a hotspot may lead to nonuniform heating as materials not present in the hotspot region are not subjected to the same degree of microwave heating [16]. Further, it was reported that the design of microwave cavity plays a crucial role in the control or utilization of this hotspot for effective heating [40]. According to a simulation study on microwave design, uneven distribution of scattered hotspots within the sample may result in non-uniform heating [16]. A major problem is faced when dealing with microwave metal-discharge applications. Here, the main obstacle is that the discharge is highly unstable and difficult to sustain for a long time [30]. In microwave systems, discharge occurs when metals with sharp edges or tips are subjected to microwave irradiations. Spark discharge at the tip of metal electrode is an example where surrounding gas is broken down to conducting ions which may lead to formation of hotspots due to excessive generation of heat in the process. Another microwave-metal discharge effect can be observed in the use of thermocouples inside a microwave field, which can cause incorrect recording of temperature and may also damage the thermocouple in the extreme case of hotspots formation. Finally, application of microwave technology is still limited to only lab scale systems and is not effective at industrial scales. This drawback can be attributed to the limited understanding of microwave systems. Apart from material properties and characteristics, operating parameters such as radiation time and power, cavity design and material throughput have been found to determine the extent to which a successful treatment is achieved [23].

\subsection{New trend in microwave assisted pyrolysis of plastics}

Literature spans through numerous works that utilized the dielectric materials as absorbents to achieve microwave assisted pyrolysis $[12,15,19,41]$. Carbonaceous materials like traditional coke, due to their excellent response to microwave absorption, heat tolerance and low cost has made them used widely in pyrolysis applications [20]. Later, in an experimental study led by Hussain et al. [42], pyrolysis was achieved effectively, based on microwave-metal interaction mechanism as a novel means of generating heat rather than the use of absorbents. Only few works have been explored principled on this mechanism [42-44]. The first experiment was conducted on a waste polystyrene (PS) plastic sample (20g), placed inside an iron mesh. The mesh acted as an antenna responsible for the generation of heat in a temperature range of $1100-1200^{\circ} \mathrm{C}$. The products obtained were $80 \%$ liquid, $15 \%$ gas and 5\% char residue [42]. In a similar work, PS waste sample was pyrolyzed using a copper coil instead of iron mesh [43]. The results gave $85 \%$ liquid, 10$12 \%$ gases and char residue. The process was achieved in a temperature range of 1000$1100^{\circ} \mathrm{C}$. Both aforementioned studies were found to be effective in producing fuel like hydrocarbons showing major content of aromatics. The use of iron mesh [42] and copper coil [43], also demonstrated that the rate of reaction and reaction time were strongly influenced by the shape and nature of metal antenna. The mechanism of heat generation is based on the repeated reflections of microwaves trapped inside the coil shaped reactor. The metal coil or mesh initially acts as an antenna for reflections. Subsequently, when there is an interaction of free or conducting electrons, the metallic coil begins to act as a microwave absorbent. The rapid vibrations of the atomic core cause volumetric and rapid heating reaching high temperatures up to the melting point of metal [43].

Though the method is suitable, it still requires extended studies to develop proper control in getting the optimum yield and specific composition of products. Also, improper control of process parameters may result in non-uniform heating that can lead to formation 
of hotspots which in turn will reduce the overall treatment efficiency and lower the effective energy utilization of microwave systems [23]. Although, the initial capital cost of microwave system is high, this can be offset by economic benefits attained in operation by process time-savings, the yield of saleable by-products and environmental compatibility [23].

\section{Conclusion}

Microwave heating technology has evolved as a smart alternative for getting selective products from the pyrolysis of plastics. The process has the potential to deliver valuable chemicals and fuels. Despite so many advantages of this technology, limitations like inaccuracy in measurement of temperature, non-uniform heating caused by scattered hotspots, limited understanding of the specific role of dielectric materials in the pyrolysis reaction and gaps in efficient design of microwave systems has constrained the successful application of microwave induced pyrolysis of plastics at industrial scale. As such further investigations are required to push the technology to higher levels of performance where scale up of the technology together with cost reduction is not an issue.

\section{References}

1. P. Lettieri and S. M. Al-Salem, Thermochemical Treatment of Plastic Solid Waste, in Waste, Elsevier, 233-242, (2011)

2. Plastics Europe, Association of Plastic Manufacturers Europe. An analysis of European plastics production, demand and waste data. Belgium: European Association of Plastics Recycling and Recovery Organisations, Plastics Europe, 132, (2015)

3. S. D. Anuar Sharuddin, F. Abnisa, W. M. A. Wan Daud, M. K. Aroua. Energy Convers. Manag., 115, 308-326, (2016)

4. U.S. Environmental Protection Agency, Common wastes and materials, U.S., (2014)

5. H. Liu, Y. Liang, D. Zhang, C. Wang, H. Liang, H. Cai. Waste Manag., 30(8-9), 1569-1576, (2010)

6. S. M. Ali, A. Pervaiz, B. Afzal, N. Hamid, A. Yasmin. J. King Saud Univ. - Sci., 26(1), 59-65, (2014)

7. B. Kunwar, H. N. Cheng, S. R. Chandrashekaran, B. K. Sharma. Renew. Sustain. Energy Rev., 54, 421-428, (2016)

8. J. Aguado, D. P. Serrano, J. M. Escola. Ind. Eng. Chem. Res., 47(21), 7982-7992, (2008)

9. R. Miandad, M. A. Barakat, A. S. Aburiazaiza, M. Rehan, A. S. Nizami. Process Saf. Environ. Prot., 102, 822-838, (2016)

10. A. Undri, L. Rosi, M. Frediani, P. Frediani. Microwave Heating, 207-232, (2011)

11. A. Undri, L. Rosi, M. Frediani, P. Frediani. Fuel, 116, 662-671, (2014)

12. K. N. Aishwarya and N. Sindhu. Procedia Technol., 25(Raerest), 990-997, (2016)

13. W.-C. Huang, M.-S. Huang, C.-F. Huang, C.-C. Chen, K.-L. Ou. Fuel, 89(9), 23052316, (2010)

14. A. López, I. de Marco, B. M. Caballero, A. Adrados, M. F. Laresgoiti. Waste Manag., 31(8), 1852-1858, (2011)

15. D. V. Suriapparao and R. Vinu. J. Anal. Appl. Pyrolysis, 113, 701-712, (2015)

16. J. P. Robinson, S. W. Kingman, C. E. Snape, S. M. Bradshaw, M. S. A. Bradley, H. Shang, R. Barranco. Chem. Eng. Res. Des., 88(2), 146-154, (2010) 
17. X. Zhang, K. Rajagopalan, H. Lei. Sustain. Energy Fuels, 0, 1-36, (2017)

18. N. M. Mokhtar, R. Omar, A. Idris. Util. Environ. Eff., 34(22), 2104-2122, (2012)

19. E. Khaghanikavkani. J. Chem. Eng. Process Technol., 4(3), (2013)

20. B. Menéndez, J. A. Arenillas, A. Fidalgo, Y. Fernández, L. Zubizarreta, E. G. Calvo, J. M. Bermúdez. Fuel Process. Technol., 91(1), 1-8, (2010)

21. K. E. Haque. Int. J. Miner. Process., 57(1), 1-24, (1999)

22. S. S. Lam and H. A. Chase. Energies, 5(10), 4209-4232, (2012)

23. T. J. Appleton, R. I. Colder, S. W. Kingman, I. S. Lowndes, A. G. Read. Appl. Energy, 81(1), 85-113, (2005)

24. E. Khanikavkani. J. Chem. Eng. Process Technol., 4(3), (2013)

25. Y. F. Huang, W. H. Kuan, S. L. Lo, C. F. Lin. Bioresour. Technol., 99(17), 8252$8258,(2008)$

26. A. Domínguez, J. Menéndez, Y. Fernández, J. J. Pis, J. M. Valente Nabais, P. J. M. Carrott, M. M. L. Ribeiro Carrott, J. Anal. Appl. Pyrolysis, 79(1-2) SPEC. ISS., 128-135, (2007)

27. Y. Fernandez, A. Arenillas, J. Angel. Advances in Induction and Microwave Heating of Mineral and Organic Materials, InTech, (2011)

28. F. Motasemi and M. T. Afzal. Renew. Sustain. Energy Rev., 28, 317-330, (2013)

29. S. Chandrasekaran, S. Ramanathan, T. Basak. AIChE J., 58(2), 330-363, (2012)

30. J. Sun, W. Wang, Q. Yue, C. Ma, J. Zhang, X. Zhao, Z. Song. Applied Energy, 175(August). Elsevier Ltd, 141-157, (2016)

31. S. Horikoshi, A. Osawa, S. Sakamoto, N. Serpone. Chem. Eng. Process. Process Intensif., 69, 52-56, (2013)

32. W. Chen, B. Gutmann, C. O. Kappe. Chemistry Open, 1(1), 39-48, (2012)

33. A. Undri, S. Meini, L. Rosi, M. Frediani, P. Frediani. J. Anal. Appl. Pyrolysis, 103, 149-158, (2013)

34. X. Wang, W. Morrison, Z. Du, Y. Wan, X. Lin, P. Chan, R. Ruan. Appl. Energy, 99, 386-392, (2012)

35. H. Lei, S. Ren, J. Julson. Energy \& Fuels, 23(6), 3254-3261, (2009)

36. N. Wang, J. Yu, A. Tahmasebi, Y. Han, J. Lucas, T. Wall, Y. Jiang. Energy \& Fuels, 28(1), 254-263, (2014)

37. D. Chen, L. Yin, H. Wang, P. He. Waste Manag., 37(12), 116-136, (2015)

38. N. I. Djeu, Fiber Optic High Temperature Sensor, United States Patent, US6045259, US6045259, (2000)

39. C. Reimbert. IMA J. Appl. Math., 57(2), 165-179, (1996)

40. D. A. Jones, T. P. Lelyveld, S. D. Mavrofidis, S. W. Kingman, N. J. Miles. Resour. Conserv. Recycl., 34(2), 75-90, (2002)

41. A. Undri, L. Rosi, M. Frediani, P. Frediani. Fuel, 133, 7-16, (2014)

42. Z. Hussain, K. M. Khan, K. Hussain. J. Anal. Appl. Pyrolysis, 89(1), 39-43, (2010)

43. Z. Hussain, K. M. Khan, K. Hussain, S. Perveen. Util. Environ. Eff., 36(18), 19821989, (2014)

44. Z. Hussain, K. M. Khan, N. Basheer, K. Hussain. J. Anal. Appl. Pyrolysis, 90(1), 53-55, (2011) 